\title{
Spectral and Color Characterization of a Quantum Dots Display for Gonio-Apparent Colors
}

\author{
Khalil Huraibat $^{1^{*}}$, Esther Perales ${ }^{1}$, Eric Kirchner ${ }^{2}$, Ivo van der Lans ${ }^{2}$, Alejandro Ferrero ${ }^{3}$, Joaquín Campos ${ }^{3}$, Francisco Miguel Martínez- \\ Verdú ${ }^{1}$
}

${ }^{1}$ Department of Optics, Pharmacology and Anatomy, University of Alicante, Alicante, Spain

${ }^{2}$ Color Research, AkzoNobel Performance Coatings, Sassenheim, the Netherlands

${ }^{3}$ Instituto de Óptica, Consejo Superior de Investigaciones Científicas (CSIC), Madrid, Spain

\begin{abstract}
Digital simulation by rendering gonio-apparent coatings and thereby visualizing the variation of visual attributes such as color and texture with geometry is requested for different industries. Recently, quantum dots (QD) displays appeared in the market with attractive characteristics such as a wider color gamut. Therefore, a proper color characterization and calibration of this type of displays is needed. In this work, we focused on the color and spectral characterization of the new quantum dots display technology. Philips Brilliance LCD Quad HD monitor was used. The spectral radiance of a set of images (stepped digital count levels from 0 to 255) was measured by the Konica-Minolta CS2000 tele-spectroradiometer. Another set of images with different combinations of digital count levels are generated for the validation. As results, a good primaries constancy was obtained after black correction. Channel additivity with errors less than 0.77 was confirmed. The electro-optical transfer function can be correctly adjusted to a power function. The GOG model was applied giving good characterization of this display. Color reproduction is evaluated using the color-difference formula CIEDE2000. The average color difference $\Delta$ Eoobetween the generated images and the objects is 1.39 for the validation generated images. Therefore, we conclude that the accuracy obtained with the GOG model is sufficient to get good performance in $3 D$ rendering. Furthermore, the color appearance of a gonio-apparent coating is reproduced on a QD display and on a sRGB display. It is checked that the $\mathrm{QD}$ display provide a more chromatic gamut, which is very useful for the color rendering of gonio-apparent coatings.
\end{abstract}

\section{Introduction}

Nowadays, digital displays and computers are widely used in visual science and visual experiments. Generally, visual stimuli are generated using software in a computer or by imaging systems before being visualized by the observers on a computer display. This makes essential the characterization of these devices to assure its reproduction reliability in terms of colors and spectral information [1].

Goniochromatic coatings have become very popular in different industrial areas like cosmetics, security inks and specially automotive [2]. The special effect is obtained by using specialeffect pigments containing metallic, interference or pearlescent pigments producing a strong color variation with the illumination/viewing geometry [3, 4]. Given this, digital simulation by rendering gonio-apparent coatings and thereby visualizing the variation of visual attributes such as color and texture with geometry is requested to well replicate and render these new colors and their appearance in a proper way, which will be of a huge help in the industrial world.

However, in a recent publication, the limitations of a conventional LCD display for this goal was shown [5], since many visual attributes of goniochromatic coatings fall outside the colorgamut of this type of electronic display. Consequently, gamutmapping algorithms [6], new extended RGB color spaces and/or multi-primaries displays are necessary to reproduce faithfully the colorimetric properties of these materials.

Recently, quantum dots (QD) displays showed up in the market with attractive characteristics such as a larger color gamut because they have narrow linewidths of emission, and high efficiency and stability. They seem to be a promising technology for vision experiments due to its numerous fundamental advantages. QD are semiconductor nanocrystals that provide vivid colors in an energy-efficient way [7,8]. The aim of this work is to evaluate the performance of a QD display for the digital color reproduction of gonio-apparent colors. Therefore, the first step is to do a proper spectral and color characterization of this device.

\section{Materials and Methods}

The display to be characterized is the Philips Brilliance LCD monitor (MultiView 272P4QPJKES Quad HD 27" / 68.6cm 2560 x 1440) available in our lab (Figure.1).

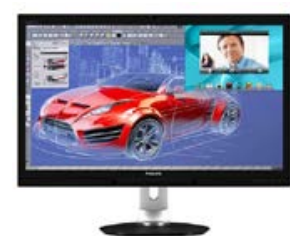

Figure 1. Philips Brilliance $L C D$ monitor with Webcam, MultiView 272P4QPJKEB Quad HD 27" (68.6 cm) 2560 x 1440 display. Source: Philips official website. 
Since the standard color display calibration method "gainoffset-gamma" (GOG) is established for CRT displays and currently is widely used in the field of vision science, a first try is carried out to check its validity for this non-CRT display $[9,10]$. This method provides the existent relation between the signal of a channel of the display and the luminance generated by this channel (electro-optical transfer function) through the equation:

$$
\begin{aligned}
& \frac{L}{L_{\max }}=\left(g_{i} N D R_{i}+o_{i}\right)^{\gamma_{i}} \quad i=R, G, B \quad \text { Eq.(1) } \\
& \text { with } \quad o_{i}+g_{i}=1
\end{aligned}
$$

where $g_{\mathrm{i}}$ and $\gamma_{\mathrm{i}}$ are the parameters to adjust the curve, $o_{\mathrm{i}}$ the offset for each channel and $N D R_{i}$ is the value of the relative or normalized digital value (ND/2 ${ }^{\text {bits }}-1$ ) of each channel: Red, green and blue, considering the depth of bits (for example, 8 bits is equivalent to 256 digital levels per channel). Therefore, conceptually, the values of $L / L \max$ for each channel RGB are equivalent in our case to the colorimetric values RGB of the display.

The transformation matrix (RGB $\rightarrow \mathrm{XYZ}$ ) can be written as:

$$
\left(\begin{array}{l}
X \\
Y \\
Z
\end{array}\right)=\left(\begin{array}{ccc}
X(R) & X(G) & X(B) \\
Y(R) & Y(G) & Y(B) \\
Z(R) & Z(G) & Z(B)
\end{array}\right)\left(\begin{array}{l}
R \\
G \\
B
\end{array}\right)
$$

where $X(R, G, B), Y(R, G, B)$ y $Z(R, G, B)$ are the tristimulus values of the primaries activated with the maximum, and the matrix RGB represents the luminance of each channel obtained considering equation (1).

For other technologies, the electro-optical transfer function does not fit in all cases to the discreet curve built for the CRTs on the GOG method [11]. On the other hand, while the physical behaviour of the other diplay technologies is different from the CRT, color mixing rules by spectral addition and luminance are the same even if the primary types are different. Therefore, depending on the display, it would be possible to characterize the electrooptical transfer function in the same way as for CRT.

For this evaluation, we need to generate a set of images to measure several luminance values of RGB. These were generated using MATLAB ${ }^{\circledR}$ (The MathWorks, Inc.) on a desktop computer where 18 different stimuli (images) are selected to cover the 8-bit grayscale values (0 to 255) for each channel. A small interval of 5 units was chosen to cover completely the low levels [0-50], while a wider interval of 25 units is selected to cover the rest of the range [50-255], the choose of a small interval in the low levels is due to the small radiance changes which allows a better representation of this problematic area. Two more stimuli were generated to simulate the white stimulus (RGB channels activated to the maximum level 255) to assess the spectral additivity and whether the spectrum of white is a linear combination of the individual components. The second simulates the dark stimulus (RGB channels inhibited, digital count level 0 ).

Luminance and spectral measurements are carried out using the tele-spectroradiometer Konica Minolta CS2000, with the help of the data management software CS-S10w. The measured values are saved in a text file to be used later for the computations on
MATLAB. Measurements are performed with a $2^{\circ}$ field of view, the objective of the tele-spectroradiometer perpendicular to the display, pointing to the center and placed at about $50 \mathrm{~cm}$ of it. Stimuli images are displayed on full screen itself in a dark room to avoid any undesired illumination from the screen. The automatic display adjustment is deactivated and fixed to the maximum brightness. The measurements output file extracted with the CSS10W contains the luminance, the chromaticity coordinates and the spectral information (from $380 \mathrm{~nm}$ to $780 \mathrm{~nm}$ ) for each stimulus.

\section{Results}

\section{Spectral characteristics}

The spectral distribution is obtained for the 18 stimuli generated on each channel separately, for the white and for the black stimuli. The spectral radiance characteristics of the display are illustrated in figures 2-3.

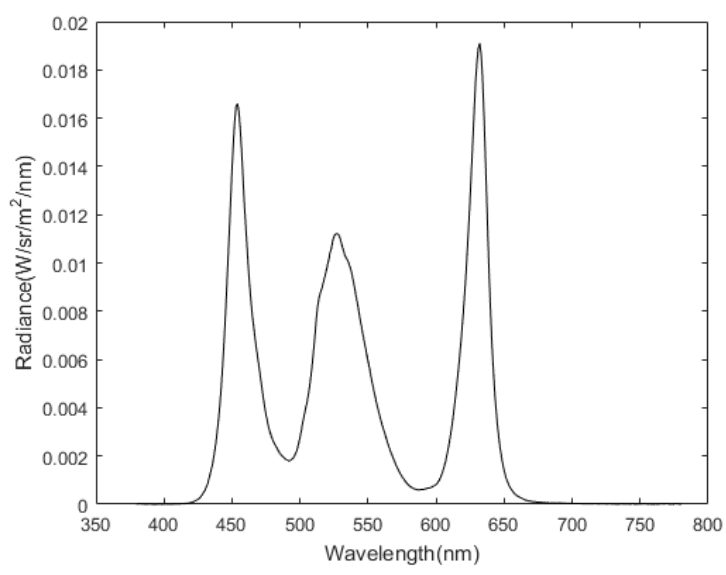

Figure 2. Spectral profile of white color.

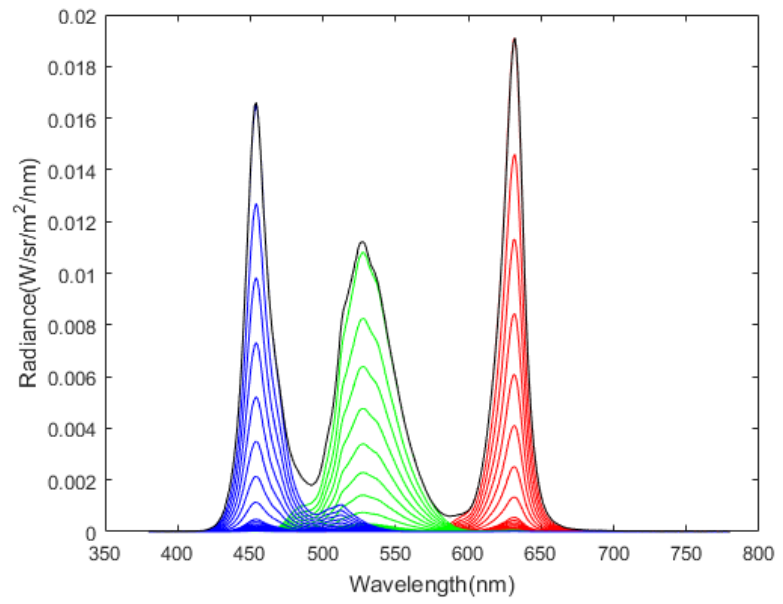

Figure 3. Spectral profiles of the RGB channels for the 18 different digital count levels at each channel separately

Figure 2 shows the spectral profile of the white on the display. The spectrum has maximum emission in three regions (454 nm, 
$527 \mathrm{~nm}$ and $632 \mathrm{~nm}$ ) and the peak spectral radiance of the white color is about $1.91 \times 10^{-2} \mathrm{Wsr}^{-1} \mathrm{~m}^{-2}$ (equivalent to a luminance of 376 $\mathrm{cd} / \mathrm{m}^{2}$ ). Figure 3 shows the spectral profile of the separate RGB primaries compared to the white (black curve). The red and blue channels spectra are narrower than the green one. The blue spectrum has a second smaller peak within the green channel region. The maximum spectrum emission matches with the white peaks for the three channels.

\section{Chromaticity constancy of primaries}

The chromaticity constancy of primaries is examined based on the measured data (xyY values) of the RGB ramps (in which each of the three primaries was stepped in digital counts from 0 to 255). Figure 4 (A) shows a chromatic representation of the measured stimuli on the CIE diagram. There is no primaries constancy as the figure shows. This can be related with the non-zero luminance of the black, therefore a correction was applied by subtracting the black luminance value to remove the effect of this undesired light [11], which leads to the following results shown on Figure 4 (B). Therefore, we can assume primaries constancy based on this correction ignoring the small aberration for the low levels, especially for the blue and red channels.
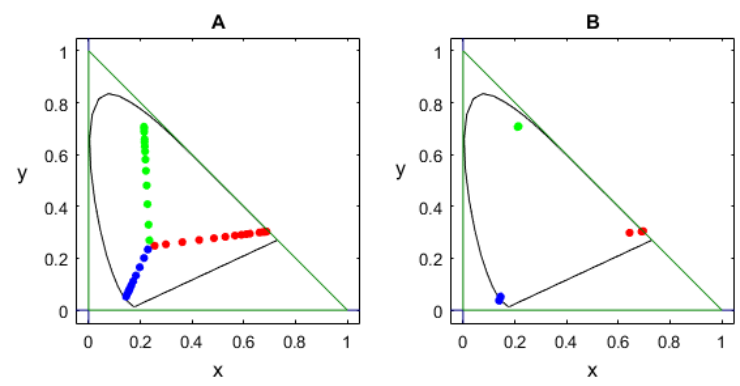

Figure 4. (A) Stimuli chromaticity representation (primaries), (B) Stimuli chromaticity representation after black correction.

\section{Additivity}

The luminance and the tristimulus additivity were examined separately. Luminance additivity is evaluated based on the measured radiance values, as well as the tristimulus values of the white against the sum of the tristimulus values of the primaries red, green and blue.

From tables (1-2) it is possible to confirm the luminance additivity as well as the tristimulus additivity. Errors are lower than $1 \%$ for all cases. Therefore, the transformation matrix mentioned before can be applied since the chromaticity constancy of primaries and additivity conditions fulfill.

Table 1. Verification of the luminance additivity.

\begin{tabular}{|c|c|}
\hline & $\mathrm{Y}\left(\mathrm{cd} / \mathrm{m}^{2}\right)$ \\
\hline $\mathrm{R}+\mathrm{G}+\mathrm{B}$ & 378 \\
\hline White & 376 \\
\hline Difference (\%) & 0.27 \\
\hline
\end{tabular}

Table 2. Verification of the tristimulus additivity.

\begin{tabular}{|c|c|c|c|}
\hline & $\mathrm{X}$ & $\mathrm{Y}$ & $\mathrm{Z}$ \\
\hline White & 337.44 & 376.42 & 442.94 \\
\hline $\mathrm{R}+\mathrm{G}+\mathrm{B}$ & 338.82 & 378.18 & 446.36 \\
\hline Difference (\%) & 0.41 & 0.47 & 0.77 \\
\hline
\end{tabular}

\section{Electro-optical transfer function}

The electro-optical transfer function describes the relationship between the signal used to drive a given display channel and the luminance produced by that channel. Figure 5 shows the luminance values $\left(\mathrm{cd} / \mathrm{m}^{2}\right)$ against the digital counts for the RGB channels separately. The curves' behavior gives a good prognostic; it seems to match with a power curve which should be evaluated using the GOG method.

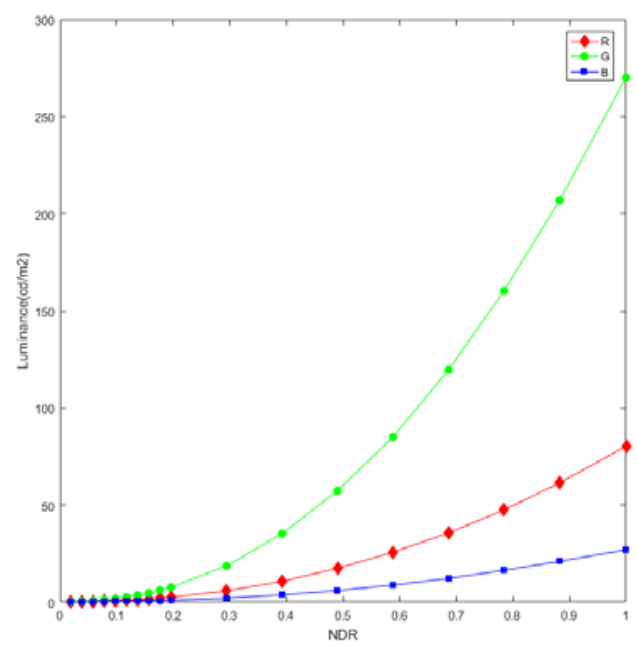

Figure 5. Luminance profile of the Quad HD Philips monitor.

Since the XYZ values obtained for the NDR $=0$ is null, and the offset value is zero due to the black correction, then, the fitting should be done for the curve:

$$
\frac{L}{L_{\max }}=\left(g_{i} N D R_{i}\right)^{\gamma_{i}} \quad i=R, G, B \quad \text { Eq. (3) }
$$

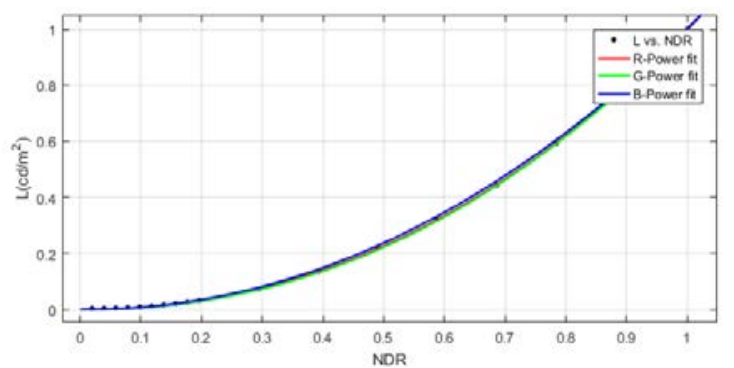

Figure 6. Power fit curves for the RGB-channel. 
Figures 6 illustrates the measured electro-optical transfer function for the three channels, which are fitted to a power function (General model Power 1).

Table 3. Power fit data for each channel.

\begin{tabular}{|c|c|c|c|c|}
\hline & \multicolumn{4}{|c|}{ QD Display } \\
\hline Channel & Gain & Gamma & $\mathrm{r}^{2}$ & RMSE \\
\hline R & 0.9989 & 2.142 & 0.999 & 0.00237 \\
\hline G & 1.002 & 2.172 & 0.999 & 0.00114 \\
\hline B & 1.002 & 2.077 & 0.999 & 0.00726 \\
\hline
\end{tabular}

\section{GOG model}

The GOG model proposed by Berns [9] could be applied to characterize this display as both conditions (additivity and power adjustment) fulfil. This matrix transformation gives the $\mathrm{XYZ}$ values from the RGB:

$$
\left(\begin{array}{l}
X \\
Y \\
Z
\end{array}\right)=\left(\begin{array}{ccc}
X(R) & X(G) & X(B) \\
Y(R) & Y(G) & Y(B) \\
Z(R) & Z(G) & Z(B)
\end{array}\right)\left(\begin{array}{l}
\left(g_{R} \cdot N D R_{R}\right)^{\gamma_{R}} \\
\left(g_{G} \cdot N D R_{G}\right)^{\gamma_{G}} \\
\left(g_{B} \cdot N D R_{B}\right)^{\gamma_{B}}
\end{array}\right) \text { Eq.(3) }
$$

The LAB values are computed for the resulting $\mathrm{XYZ}$ and for the measured values (xyY from the tele-spectroradiometer). CIEDE2000 color-difference formula was used to compare the reproduced colors with the generated calibration stimuli. The average of $\Delta \mathrm{E}_{00}$ is 1.06 .

In the same way, a proper evaluation process is carried out by applying the resulting method on 16 different images generate with different combinations of digital count levels, where $\Delta \mathrm{E}_{00}$ between the measured and computed $\mathrm{LAB}$ values after applying the method is computed. The resulting $\Delta \mathrm{E}_{00}$ confirms the validity of the applied calibration method where the average $\Delta \mathrm{E}_{00}$ for the 16validation stimulus is of 1.39 (Table 4).

Table 4. CIEDE200 color difference between measured and computed $L^{*} a^{*} b^{*}$ values of validation stimulus.

\begin{tabular}{|l|l|}
\cline { 2 - 2 } \multicolumn{1}{c|}{} & $\Delta \mathrm{E}_{00}$ \\
\hline Maximum $\Delta \mathrm{E}_{00}$ & 3.08 \\
\hline Minimum $\Delta \mathrm{E}_{00}$ & 0.22 \\
\hline Averaged $\Delta \mathrm{E}_{00}$ & 1.39 \\
\hline
\end{tabular}

Another evalulation is performed to test whether the color gamut of the Philips Brilliance LCD Quad HD monitor verifies the ITU-R Recommendation BT.2020 color space[12]. Table 5 illustrates the numerical differences between the color coordinates of the primeries and the white of our display comparing to the standards of Rec. 2020.
Table 5. Validation of the BT. 2000 on the Philips Brilliance LCD Quad HD monitor.

\begin{tabular}{|c|c|c|c|c|c|c|c|c|}
\hline \multirow{2}{*}{$\begin{array}{c}\text { Color space } \\
\text { and gamut }\end{array}$} & \multicolumn{2}{|c|}{ White point } & \multicolumn{6}{|c|}{ Primary colors } \\
\cline { 2 - 9 } & $x_{W}$ & $y_{W}$ & $x_{R}$ & $y_{R}$ & $x_{G}$ & $y_{G}$ & $x_{B}$ & $y_{B}$ \\
\hline $\begin{array}{c}\text { ITU-R } \\
\text { BT.2020 }\end{array}$ & 0.31 & 0.33 & 0.71 & 0.29 & 0.17 & 0.80 & 0.13 & 0.05 \\
\hline $\begin{array}{c}\text { PHILIPS } \\
\text { QuadHD }\end{array}$ & 0.29 & 0.32 & 0.69 & 0.30 & 0.21 & 0.71 & 0.14 & 0.05 \\
\hline $\begin{array}{c}\text { Difference } \\
(\%)\end{array}$ & 7.26 & 2.74 & 2.54 & 2.74 & 23.53 & 10.92 & 6.87 & 13.04 \\
\hline
\end{tabular}

After computing the difference between both color gamuts, we obtained that the Philips Brilliance LCD Quad HD monitor color gamut covers $81 \%$ of the Rec.2020 color space (Figure 7).

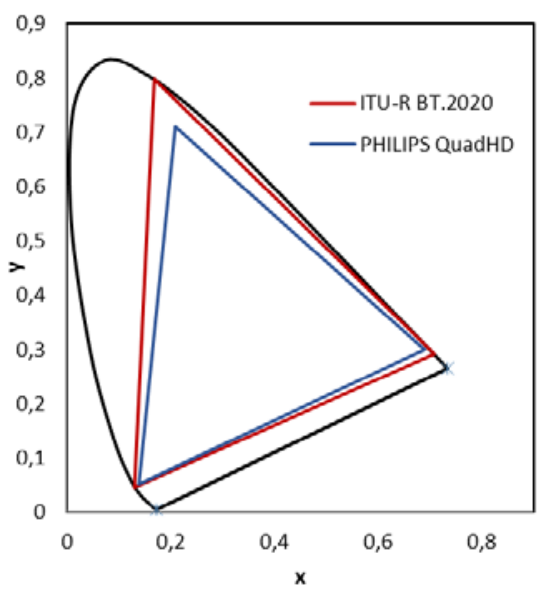

Figure 7. CIE 1931 chromaticity diagram showing the triangles and the location of the primary colors of the ITU-R BT.2020 and the PHILIPS QuadHD displya

\section{Color reproduction of effect coatings}

In a previous work, it was found that for many geometries, the resulting colors lie outside the color-gamut of an electronic display[13]. Therefore, it was evaluated the color gamut of this QD display in terms of the ability to replicate or to render the color appearance of effect coatings. A special effect coating (Light Blue \& Pale Turquoise) was considered to then be compared with the color gamut of the QD display. The sample was measured by the gonio-spectrophotometer developed at the Instituto de Óptica in CSIC (IO-CSIC) at any geometry, including out-of-plane and retroreflection angles following a normalized procedure[14]. In Figure 10, CIELAB diagrams are shown. The color dots are the samples associated with each measurement geometry with the projection on the CIE a*-b* diagram by grey dots. The color gamuts of the QD display (left) and a sRGB LCD display (right) are plotted by constant lightness profiles (from $L^{*}=0$ to $L^{*}=100$ in steps of 5) for better visualization. 

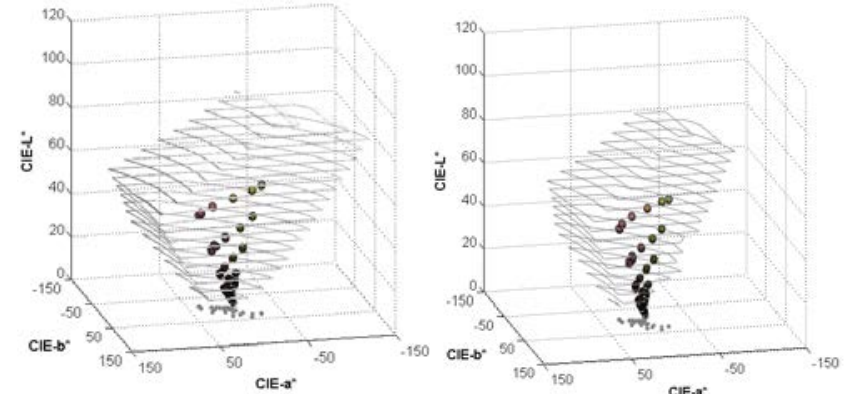

Figure 8. Three-dimensional CIELAB diagrams for a gonio-apparent coating (colorstream) comparing with the QD color gamut (left) and SRGB color gamut (right).

As it can be seen in Figure 8, the color gamut associated with the QD display is greater than for the sRGB display. From this graph, it is difficult to visualize the digital color reproduction of the gonio-apparent coating in each display. For this reason, Figure 9 shows the CIE-a*b* diagrams for two different lightness profiles $\left(L^{*}=40\right.$ and $\left.L^{*}=45\right)$. In these graphs, the great difference between both displays is observable. In fact, some color stimuli of the gonio-apparent coating cannot be reproduced in the sRGB display, while the QD display could reproduce them since its color gamut includes chromatic colors.
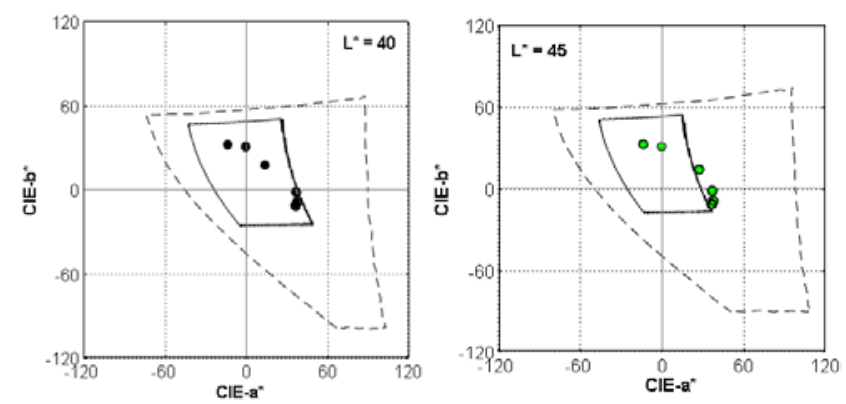

Figure 9. CIE-a* $b^{*}$ diagrams for a gonio-apparent coating (colorstream) comparing with the $Q D$ color gamut and SRGB color gamut for two different lightness profiles. The dashed line corresponds to the QD display color gamut and the solid line to the SRGB display color gamut.

\section{Conclusions}

As results, a good chromaticity constancy of primaries was obtained after black correction. It was checked that this display satisfies channel additivity with errors smaller than 0.77 and the electro-optical transfer function for the three channels can be modelled as a power function. The GOG model was found to characterize well this display. Color reproduction was evaluated in terms of CIEDE2000. The computed CIELAB values of a set of images differ from their objects' coordinates by an average value of $\Delta \mathrm{E}_{00}=1.39$. Therefore, we conclude that the accuracy obtained with the GOG model is sufficient to get good performance in 3D color rendering. On the other hand, it is proved that the QD display can render more chromatic colors than SRGB LCD displays since its primaries are spectrally narrower what is very useful for color rendering of gonio-apparent coatings.

\section{Acknowledgements}

We would like to thank the Ministry of Economy and Competitiveness for the project DPI2015-65814-R. Khalil Huraibat would also like to thank the Ministry of Economy and Competitiveness for his pre-doctoral fellowship grant (FPI BES2016-077325).

\section{References}

[1] J., Brucker, CRT Characterization and Metameric Color Reproduction. Department of Physics, University of Joensuu, 2004.

[2] A., Ferrero, et al., "Measuring and specifying goniochromatic colors". 23rd ICO conference, Santiago de Compostela 2014.

[3] G., Pfaff, "Special Effect Pigments, in High Performance Pigments". 2009, Wiley-VCH Verlag GmbH \& Co. KGaA. p. 75-101.

[4] F.J., Maile, G. Pfaff, and P. Reynders, "Effect pigments-past, present and future”. Progress in organic coatings, 2005. 54(3): p. 150163.

[5] Cooper, E.A., et al., “Assessment of OLED displays for vision research”. Journal of Vision, 2013. 13(12): p. 16-16.

[6] E., Kirchner, et al. "Fast And Accurate 3D Rendering Of Automotive Coatings”. in Color and Imaging Conference. 2015. Society for Imaging Science and Technology.

[7] J.S., Steckel, et al., "Quantum dots: The ultimate down-conversion material for LCD displays”. Journal of the Society for Information Display, 2015. 23(7): p. 294-305.

[8] P., Kathirgamanathan, et al., "Electroluminescent organic and quantum dot LEDs: The state of the art”. Journal of Display Technology, 2015. 11(5): p. 480-493.

[9] R.S., Berns, "Methods for characterizing CRT displays". Displays, 1996. 16(4): p. 173-182.

[10] G., Sharma, Digital Color Imaging Handbook. Electrical Engineering and Applied Signal Processing Series. 2003: CRC press.

[11] B., Bastani, B. Cressman, and B. Funt, "Calibrated color mapping between LCD and CRT displays: A case study”. Color Research \& Application, 2005. 30(6): p. 438-447.

[12] I.T., Union, Recommendation ITU-R BT.2020-2. Electronic Publication, 2015.

[13] E., Perales, et al. "Color gamut of a typical display for the color reproduction of effect coatings". in CONGRESS OF THE INTERNATIONAL COMMISSION FOR OPTICS, Santiago de Compostela. 2014.

[14] A., Rabal, et al., “Automatic gonio-spectrophotometer for the absolute measurement of the spectral BRDF at in-and out-of-plane and retroreflection geometries”. Metrologia, 2012. 49(3): p. 213.

\section{Author Biography}

Khalil Huraibat received his BSc in Optics and Optometry from the University of Granada, Spain, in 2014, and his MSc under the Erasmus Mundus program "Color in Informatics and Media Technology (CIMET)" from both universities, Granada, Spain, and Jean Monet of Saint Etienne, France, from 2014 to 2016. He is doing his PhD in Applied Physical Sciences and Technologies in the University of Alicante since the beginning of 2017. His research interests include applied Color Science and Technology, gonio-appearance of materials and $3 D$ printing. He is current member of the Color \& Vision Group (http://web.ua.es/en/gvc) of the University of Alicante. 